\title{
LOCALLY PERIPHERALLY EUCLIDEAN SPACES ARE LOCALLY EUCLIDEAN
}

\author{
BY O. G. HARROLD, JR. ${ }^{1,2}$ \\ Communicated by R. H. Bing, January 17, 1960
}

In this paper an outline is given of a characterization among the Peano spaces (i.e., among the compact, locally connected continua) of those spaces that are locally euclidean in dimension 3 . A characterization of the 3-sphere among Peano spaces has been given by R. H. Bing [1] and it is his result together with the notion of a "columnar partitioning" that enables one to characterize the locally euclidean spaces.

Briefly, a partitioning of a space $S$ is a finite collection of mutually exclusive connected open sets whose union is dense in $S$. A sequence $G_{1}, G_{2}, \cdots$ of partitionings of a space is called a decreasing sequence of partitionings if $G_{i+1}$ is a refinement of $G_{i}$ (i.e., an element of $G_{i+1}$ is a subset of some element of $G_{i}$ ) and the maximum of the diameters of the elements of $G_{i}$ approaches 0 with $1 / i$. A partitioning is regular if each of its elements is the interior of the closure of this element.

A necessary and sufficient condition that a Peano space $X$ be a topological 3-sphere is that one of its decreasing sequences of regular partitionings have the following four properties:

(1) The boundary of each element of $G_{i}$ is a simple closed surface.

(2) If the boundaries of two elements in $U G_{i}$ meet, the intersection is a 2 -cell.

(3) The intersection of the boundaries of three elements of $G_{i}$ is 1-dimensional at each of its points.

(4) If $g$ is an element of $G_{i-1}$ and $F(g)$ its boundary ( $g=X$ if $i=1$ ), then the elements of $G_{i}$ in $g$ may be ordered $g_{1}, \cdots, g_{n}$ so that

$$
F\left(g_{j}\right) \cap\left\{F(g) \cup F\left(g_{1}\right) \cup \ldots \cup F\left(g_{j-1}\right)\right\}
$$

is connected, $j=1, \cdots, n$ (provided $i>1$ ).

The notations are generally those of set-theory. The null set is denoted by $\square$. The diameter of a set $A$ is denoted by $\delta(A)$.

Columnar Partitionings. A regular partitioning is a brick partitioning if each element of $G$ is uniformly locally connected and if the interior of the closure of the union of any two elements is uniformly locally connected. An example of a brick partitioning of a spherical surface is a decomposition into the interiors of (spherical) hexagons.

${ }^{1}$ John Simon Guggenheim Memorial Fellow.

2 Research also supported in part by NSF G-8239. 
On the other hand, the collection of open 2-simplices corresponding to a simplicial sub-division of a spherical surface is not a brick partitioning.

The brick partitionings of a spherical surface that we shall find most convenient are those such that the elements are related locally as are the hexagons decomposing the plane with the additional requirement that the intersection of the closures of two elements be null or connected.

Suppose $S$ is a 2 -sphere and $h_{1}, \cdots, h_{n-1}$ a brick partitioning of $S$ as above. Let $U$ be an open set with boundary $S$ and $g_{1}, \cdots, g_{n}$ a partitioning of $U$ such that

$$
\begin{aligned}
& F\left(g_{i}\right) \text { is a topological } 2 \text {-sphere; } \\
& F\left(g_{i}\right) \cap F\left(g_{j}\right)=\square \text { or a disk according } \\
& \text { as } \bar{h}_{i} \cap \bar{h}_{j}=\square \text { or an arc, } \\
& F\left(g_{i}\right) \cap S=\bar{h}_{i}, \quad i<n, \\
& F\left(g_{i}\right) \cap F\left(g_{n}\right) \text { is a disk; } \\
& F\left(g_{i}\right) \cap F\left(g_{j}\right) \cap F\left(g_{k}\right)=\square \text { or an arc. }
\end{aligned}
$$

Let $\mathcal{C}$ be the partitioning of $U$ by $g_{1}, \cdots, g_{n}$ corresponding to the brick partitioning $B=\left\{h_{1}, \cdots, h_{n-1}\right\}$ of $S$. Then $\mathcal{C}$ is a columnar partitioning of $U$ determined by $B$. From (b) and (c) above it follows that two elements $g_{i}, g_{j}(i, j,<n)$ of $\mathfrak{e}$ have a non-null intersection of their closures if and only if $h_{i}$ and $h_{j}$ of $B$ have a non-null intersection of their closures. It turns out that for the class of spaces studied below that $\bar{g}_{i}$ is a topological product $\bar{h}_{i} \times I, I=[0,1]$.

If $h_{i}^{\prime}=F\left(g_{n}\right) \cap F\left(g_{i}\right)$, then $h_{1}^{\prime}, \cdots, h_{n-1}^{\prime}$ give a partitioning of $S^{\prime}=F\left(g_{n}\right)$ that is isomorphic to the partitioning $h_{1}, \cdots, h_{n-1}$ of $S$. Taking $U^{\prime}=g_{n}$ the process is iterated to find a columnar partitioning of $U^{\prime}$.

In order to obtain conditions (2) and (3) of Bing's theorem the partitioning of $U^{\prime}$ cannot be generated by the decomposition $\left(h_{1}^{\prime}, \cdots, h_{n-1}^{\prime}\right)$ of $S^{\prime}$. Hence we start from a simplicial subdivision $\mathscr{K}_{1}$ of $S^{\prime}$ such that a 2 -simplex of $\mathscr{K}_{1}$ and $\bar{h}_{i}^{\prime}$ meet in a disk or the null set. From $\mathfrak{K}_{1}$ one derives a brick partitioning $\Theta^{*}$. Then $\Theta^{*}$ induces a columnar partitioning $\mathfrak{e}^{*}$ of $U^{\prime}$. The elements of $\mathfrak{e}^{*}$ and those elements of $\mathfrak{e}$ not in $g_{n}$ give a brick partitioning of $U$.

The advantage of dealing with a "columnar partitioning" of $U$ is that it enables one rather easily to satisfy the ordering condition (4) above. By iteration one arrives at a "layer of columnar partitions."

Properties of the family $S$. The Peano space $X$ under consider- 
ation is assumed to have at each point a local basis of open sets whose boundaries are in the family $\delta$ defined as follows:

1. Separation Axiom. Each $S \in \mathcal{S}$ is a topological 2-sphere that separates $X$ irreducibly.

2. UNION AXIOM. If $D_{1}, D_{2}$ are disks, each a subset of some element of $\mathcal{S}$, and if $D_{1} \cap D_{2}$ is a nondegenerate connected subset of the intersection of their boundaries, then an element of $\mathcal{S}$ exists that contains $D_{1} \cup D_{2}$.

3. Deformation axiom. If $D$ is a disk, $D \subset S, S \in \mathcal{S}, U$ a component of $X \backslash S, V$ an open set, $V \supset \operatorname{Int} D$, then there is an isotopy, the identity on $X \backslash V$ such that $F_{t}($ Int $D) \subset U$ for $0<t$, where $F_{t}(x)$, $0 \leqq t \leqq 1$, is a function realizing the isotopy.

4. Contraction Axiom. There is a number $\eta, 0<\eta<1$, such that if $S \in \mathcal{S}, U$ a component of $X \backslash S$ and $B$ a brick partitioning of $S$ of sufficiently small mesh, then there is a layer of columnar partitions of $U$ into sets $g_{1}, \cdots, g_{n}$ generated by $B$ whose boundaries are elements of $S$ and a homeomorphism $t$ of $\bar{U}$ on $\bar{U}$, the identity on $S$ such that if $g_{i}^{\prime}=t\left(g_{i}\right)$, then

$$
\delta\left(g_{i}^{\prime}\right) / \delta(U)<\eta
$$

5. MAXimality AXIOM. If $s^{\prime}$ is another system of sets satisfying $1-4$, then $s^{\prime} \subset s$.

Theorem A. Let $X$ be a Peano space such that each point has a local basis consisting of open sets whose boundaries are elements of $\mathcal{S}$, then $X$ is a topological 3-sphere.

LOCALLY EUCLIDEAN SPACES AND THE FAMILY $\mathcal{S}^{\prime}$. Let $\boldsymbol{S}^{\prime}$ be a family of sets satisfying conditions similar to conditions 1-5 that define $\mathcal{S}$. Condition $1^{\prime}$ for $S^{\prime}$ reads as condition 1 for the family $S$ except that it is required to hold only for sufficiently small sets $S$. Condition $4^{\prime}$ for the family $\mathcal{S}^{\prime}$ reads as condition 4 but is required to apply only if $S$ is sufficiently small so that $1^{\prime}$ holds and the condition on $U$ is imposed only for the small component of $X \backslash S$.

The other conditions on $\mathcal{S}^{\prime}$ are identical with the corresponding conditions on $S$.

Theorem B. If $X$ is a Peano space such that each point has a local basis consisting of open sets whose bounaries are elements of $\mathcal{S}^{\prime}$, then $X$ is a topological 3-manifold.

TheOREM C. If $\mathcal{S}$ is the family of tame 2-spheres in the topological 3-sphere, $S^{3}$, then $S$ satisfies the Axioms 1-5. 
TheOREM D. If $X$ is a compact, closed topological 3-dimensional manifold, then $X$ is a 3-sphere if and only if each tame 2-sphere in $X$ satisfies the Separation and Contraction Axioms for the family s.

The details of the proofs of the above theorems will appear elsewhere.

\section{REFERENCE}

1. R. H. Bing, $A$ characterization of 3-space by partionings, Trans. Amer. Math. Soc. vol. 70 (1951) pp. 15-27.

University of Tennessee 